\title{
PEI asks residents how to improve health
}

$\mathrm{W}$

ith worse health indicators than Canada as a whole, plus rising levels of alcoholism and child obesity, Prince Edward Island has turned to residents for suggestions on how to improve its public health programs and policies.

The Department of Health and Wellness is now evaluating the results of its online Wellness Strategy survey, which asked islanders to respond to multiplechoice questions and provide comments on how the province could best promote positive mental health, physical activity, healthy eating, tobacco-free living and responsible alcohol consumption. When the online survey closed at the end of January, 500 people had completed it and dozens had sent written responses.

"People were really appreciative of the opportunity to have input," says John Morrison, director of sport, recreation and healthy living at the Department of Health Wellness.

The province is embarking on a new "Culture of Wellness" strategy after a 2012 report held some startling revelations. Although smoking rates had decreased, $26 \%$ of residents reported heavy drinking in 2009-2010, compared with $23 \%$ in 2001. In addition, $6.4 \%$ of children were obese, compared with $5.3 \%$ in 2005 and a Canadian rate of $4.4 \%$. Among adults, $21.7 \%$ are obese, compared with a Canadian average of $18.1 \%$.

Prince Edward Island lagged behind the rest of Canada in other areas as well. For example, $37 \%$ of people above age 12 reported consuming fruits or vegetables five or more times per day, below the Canadian rate of $44 \%$.

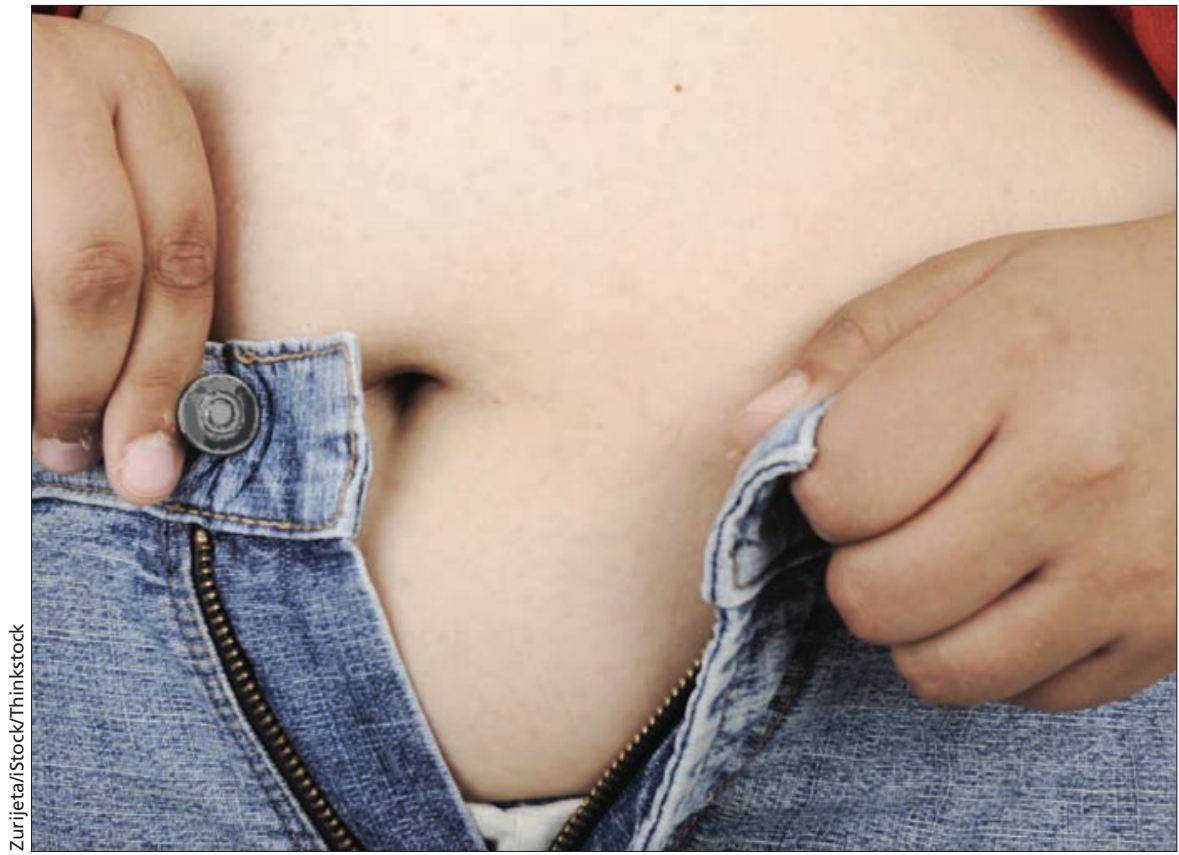

Prince Edward Island's obesity rates among children and adults are higher than the Canadian average.

"People point at things like our climate," says Morrison. "Maybe it's income levels, literacy levels. There are probably many factors contributing to why the numbers appear higher at times."

The feedback highlighted the importance of doing more for the senior population, as well as improving access to rural areas, says Morrison. Respondents also called for continued funding and expansion of many existing programs, which Morrison says is particularly helpful. "We're not always great at monitoring and evaluating these things on a dayto-day basis." Go! PEI, one of the programs survey respondents advocated for, offers free recreational activities and healthy-eating classes in communities across the island.

But islanders expressed wariness about increasing taxes on items like cigarettes, for example. "Don't do something that will hurt those who are struggling to get by," was a common response, according to Morrison.

The department will begin to implement programs or policies that have short-term impacts, before developing longer-term strategies "to let Islanders know we've heard from them." Wendy Glauser, Toronto, Ont.

CMAJ 2014. DOI:10.1503/cmaj.109-4746 